\title{
EDITORIAL \\ Peer Review
}

Enoka Corea (Dhttp://orcid.org/0000-0003-1450-2098, Himani Molligoda (iDhttp://orcid.org/0000-0002-9216-9035

Co-Editors

\section{Keywords: peer review}

(C) Authors. This is an open-access article distributed under a Creative Commons AttributionShare Alike 4.0 International License (CCBY-SA 4.0), which permits unrestricted use, distribution, and reproduction in any medium, provided the original author and source are attributed and materials are shared under the same license.

The aim of medical research is to generate new knowledge that will lead to improvements in health care and the wellbeing of people. For medical research to fulfil this aim it should be valid and reliable. The reliability of research is judged at various points in time; review by institutional research committees, grant organizations, ethics review committees etc. However, the most important appraisal or quality assurance of research takes place when the completed research is put forward for publication, during the process of journal peer review.

Peer review is where the authors' research is subjected to scrutiny by experts in the same field ('peers') before it is published. The peer 'expert' is considered to be independent of the authors and the editors and provides feedback to them, including his/her opinion on whether the research is of good quality and worthy of publication. It is expected that peer review will exclude poor research and improve and clarify good research. It is assumed that peer review is objective, fair and rational and that articles published following peer review are trustworthy. Information contained in such published papers is, then, incorporated into the current body of scientific knowledge and is used as evidence for clinical decision making.

Peer review is a vital component of the research and publication process. However, the impact of peer review is difficult to measure and systematic reviews on its effects have been inconclusive [1]. Peer review is still, largely, an ad hoc process, prone to many flaws. What are the criteria for a peer? Is it expertise in the area? In methodology? In statistics? Or all three? There is currently no training, qualification or standard for peer review. Any scientist, who is an expert in the area of the research is assumed to have the competence to review and evaluate the paper. How rigorous should the review process be? For example, should the reviewer repeat all the data analyses and read all the references? The fact that peer review is anonymous and is not subject to quality review compounds the problem.

It has been shown that peer review can be highly subjective, inconsistent and prone to bias [2]. Two peers may give conflicting and contradictory reviews. Bias against authors from less well-known institutions and against studies with negative outcomes have been 
noted [2]. Peer review increases the delay and costs of publication. It takes up the time of academics that may be better spent on further research.

Many efforts have been taken to improve the peer review process. As far back as 2007, Irene Hames published a book dedicated to peer review and manuscript management which spanned the whole gamut of publication ethics [3]. Appendix 1 of the book was republished in Science Editing in 2016 [4]. Many journals and organisations have tried to systematize peer review and make it more transparent. Reviewing protocols and checklists are provided by some journals [5]. Some journals have attempted training reviewers. Providing feedback and rewards to reviewers are increasingly practiced. However, no uniform system of standardized peer review is universally recognized or adopted.

In conclusion, it is noted that peer review remains essential to science and is widely accepted by the scientific community. In most cases, peer review is done as a voluntary service to improve science. However, the lack of any evidence of its effectiveness underlines the essentially unscientific nature of the current practice. There is a need to identify the aims and objectives of peer review and formulate a list of core reviewer competencies that can be used to train potential reviewers and monitor and evaluate reviewer quality $[6,7]$.

\section{References}

1. Jefferson T, Alderson P, Wager E, Davidoff F. Effects of Editorial Peer Review. A Systematic Review. JAMA. 2002;287(21):2784-2786. https://doi.org/10.1001/jama.287.21.2784

2. Smith R. Peer review: a flawed process at the heart of science and journals. Journal of the Royal Society of Medicine. 2006;99(4):178-182. https://doi.org/10.1177/014107680609900414

3. Hames I. Peer review and manuscript management in scientific journals: guidelines for good practice. Malden Blackwell Publishing 2007. https://doi.org/10.1002/9780470750803

4. Hames, Irene. (2016). Peer review golden rules and good practice checklist. Science Editing. 3. 36-42. https://doi.org/10.6087/kcse.61

5. Hirst A, Altman DG: Are peer reviewers encouraged to use reporting guidelines? A survey of 116 health research journals. PloS One. 7:e35621. https://doi.org/10.1371/journal.pone.0035621

6. Warne V. Rewarding reviewers - sense or sensibility? A Wiley study explained. Learn Publ. 2016;29(1):41-50. https://doi.org/10.1002/leap.1002

7. Patel J. Why training and specialization is needed for peer review: a case study of peer review for randomized controlled trials. BMC Medicine. 2014;12:128. doi:10.1186/s12916-014-0128- 